Conclusion In children with malnutrition BMI, MUAC, TST and low serum albumin levels correlated with genotype GG and CG of the IL-6 572 gene.

\section{PS-078 CLINICAL RELEVANCE OF GAMMA-GLUTAMYL TRANSPEPTIDASE IN CHILDHOOD OBESITY}

${ }^{1} \mathrm{P}$ Codoñer-Franch, ${ }^{1} \mathrm{M}$ Salamanca, ${ }^{2} \mathrm{~A}$ Codoñer-Alejos, ${ }^{1} \mathrm{M}$ Porcar-Almela, ${ }^{1} \mathrm{M}$ Navarro-Solera,

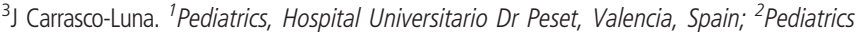
Obstetrics and Gynecology, University of Valencia, Valencia, Spain; ${ }^{3}$ Experimental Sciences, Catholic University of Valencia, Valencia, Spain

\subsection{6/archdischild-2014-307384.375}

Background and aims Metabolic risk leads to severe comorbidities in obesity. We evaluate the relationship between the values of gamma-glutamyl trans peptidase (GGT), a marker of hepatic involvement, and cardio metabolic risk factors in obese children. Methods A prospective cross-sectional study of 147 children (aged 7 to 16 years) was carried out. Ninety-five children were obese with a body mass index standard deviation score (SDS-BMI) $>2$ and 52 children were normal weight. Patients with endocrine disease or syndromic obesity were excluded. We have analysed clinical parameters of adiposity (fat mass by bioelectrical impedance, waist and hip circumference), blood pressure, and classical biochemical parameters indicative of metabolic risk (lipid profile, glucose and insulin). Additionally, novel parameters related to metabolic risk such as uric acid, retinol binding protein (RBP4), cystatinC, homocysteine, thyrotropin, ultrasensitive C-reactive protein (CRP) and GGTwere also determined. Statistical analysis was made ANCOVA test and Pearson partial correlation adjusting for gender, age, Tanner stage, and BMI.

Results GGT was higher in the children with SDS-BMI $>4$ with respect children with SDS-BMI between 2 and $4(16.3 \pm 5.8 v \mathrm{~s}$ $18.4 \pm 8.8 \mathrm{IU} / \mathrm{L}, \mathrm{p}=0.025)$. Both groups were statistically significant with respect normal weight $(12.2 \pm 2.9 \mathrm{IU} / \mathrm{L}, \mathrm{p}<$ 0.0001 and $\mathrm{p}<0.001$ respectively). GGT was correlated with SDS-BMI ( $p<0.0001)$, waist circumference ( $p<0.001)$, percentage of fat mass $(p<0.01)$, SDS of systolic blood pressure ( $p$ $<0.010)$, total cholesterol ( $\mathrm{p}<0.0001)$, LDL cholesterol $(\mathrm{p}<$ $0.0001)$, triglycerides $(\mathrm{p}<0.0001)$, RBP4 $(\mathrm{p}<0.047)$, thyrotropin $(\mathrm{p}<0.019)$ and CRP $(\mathrm{p}<0.044)$.

Conclusion GGT is a marker associated with several metabolic risk factors, which highlights the importance of considering hepatic impairment as a component of this syndrome.

\section{PS-079 WITHDRAWN}

\section{PS-080 PREVALENCE OF OVERWEIGHT IN PAEDIATRIC INFLAMMATORY BOWEL DISEASE IN SAUDI ARABIA}

${ }^{1} \mathrm{M}$ El Mouzan, ${ }^{2} \mathrm{~A}$ Mehaidib, ${ }^{3} \mathrm{M}$ Hasosah, ${ }^{4} \mathrm{~A}$ Anazi, ${ }^{5} \mathrm{~A}$ Al Hussaini, ${ }^{6} \mathrm{~K}$ Nouli, ${ }^{7} \mathrm{~K}$ Al Reheili. ${ }^{1}$ Pediatrics, King Saud University, Riyadh, Saudi Arabia; ${ }^{2}$ Pediatrics, King Faisal Specialist Hospital and Research Center, Riyadh, Saudi Arabia; ${ }^{3}$ Pediatrics, National Guard Hospital, Jeddah, Saudi Arabia; ${ }^{4}$ Pediatrics, King Fahad Specialist Hospital, Dammam, Saudi Arabia; ${ }^{5}$ Pediatrics, King Fahad Medical City, Riyadh, Saudi Arabia; ${ }^{6}$ Pediatrics, Dhahran Health Center, Dhahran, Saudi Arabia; ${ }^{7}$ Pediatrics, Maternity and Children Hospital, Madinah, Saudi Arabia

10.1136/archdischild-2014-307384.376

Background and aim Excess weight in inflammatory bowel disease (IBD) represents an additional morbidity, and yet the prevalence has been rarely reported. The aim of this report is to establish the prevalence of overweight in children with IBD in the Kingdom of Saudi Arabia (KSA).

Methods Data from a cohort of children in the KSA diagnosed with IBD were analysed retrospectively. Growth parameters were recorded at diagnosis and body mass index (BMI) was calculated using the formula (weight/height ${ }^{2}$ ). The KSA charts were used as reference. Excess weight categories were defined as overweight (BMI-for age $\geq 85$ th to $<95$ th), obesity $\geq 95$ th to $<97$ th), and severe obesity $\geq 97$ th percentile. Chi-square test was used and $p$ value of $<0.05$ was considered significant.

Results There were 417 children from birth to 18 years of age, including 133 ulcerative colitis (UC) (32\%), and 284 Crohn disease (CD) (68\%). The prevalence of excess weight was $12 / 133$ $(9 \%)$ in UC and $23 / 284(8.1 \%)$ in CD ( $p=0.063)$ much lower than in Western reports. However, the more common prevalence of excess weight in UC than $\mathrm{CD}$, although not significant $(\mathrm{p}=$ 0.063), was similar to patterns from other population. The commonest form of excess weight was overweight 20/35 (57\%), followed by obesity 9/35 (26\%), and severe obesity 6/35 (17\%).

Conclusion The pattern of excess weight in KSA children with IBD is similar to Western literature. However, a much lower prevalence is demonstrated. Identification of factors associated with the low prevalence of overweight and obesity is needed.

\section{PS-081 LACTOBICILLUS ACIDOPHILUS ATTENUATED SALMONELLA-INDUCED INTESTINAL INFLAMMATION VIA TGF-BETA/SMADS SIGNALLING}

${ }^{1}$ IF Huang, ${ }^{2}$ YC Liu, ${ }^{2}$ PF Liu, ${ }^{2}$ CW Shu. 'Pediatrics, Kaohsiung Veterans General Hospital, Kaohsiung, Taiwan; ${ }^{2}$ Medical Education and Research, Kaohsiung Veterans General Hospital, Kaohsiung, Taiwan

\subsection{6/archdischild-2014-307384.377}

Aims To investigate whether probiotics and/or prebiotics attenuate Salmonella typhimurium induced NF- $\kappa \mathrm{B}$ activation via Smad7 and $\mathrm{I} \kappa \mathrm{B} \alpha$ expression in the human colorectal epithelial $\mathrm{CaCO}_{2}$ cells; to determine the molecular mechanisms of preventive effects of probiotics on intestinal infection.

Material and methods $\mathrm{CaCO}_{2}$ cells were administered probiotic (Lactobicillus acidophilus) and/or prebiotic (inulin supplemented with oligofructose). Subsequently, the cells were infected with $S$. typhimurium. The culture supernatants and cell lysates were collected for cytokine determination and western blot analysis. The $\mathrm{CaCO}_{2}$ cells were also transfected with plasmids containing Smads or NF- $\kappa$ B responsive reporter luciferase. After transfection, supernatants from cells were collected for luciferase assay. Involvement of miR-21 (Smad7 silencer) from supernatants of infected cells in the presence or absence of probiotics was determined.

Results The probiotics significantly suppressed NF- $\kappa$ B activation elevated by $S$. typhimurium. IL-8 mRNA was significantly lower in probiotics pretreated $\mathrm{CaCO}_{2}$ cells compared with the cells infected with $S$. typhimurium alone. Synbiotics showed strongly suppressed effects on IL- 8 and TNF- $\alpha$ gene transcriptions elevated by $S$. typhimurium. Pretreatment of probiotics increased $\mathrm{I} \kappa \mathrm{B} \alpha$ expression level. Consistent with $\mathrm{I} \kappa \mathrm{B} \alpha$ expression, pretreatment of probiotics increased 7 folds of $S \operatorname{mad} 3 / 4$ activity. The protein expressions of TGF- $\beta$ and Smad7 in S. typhimurium infected cells with or without probiotics were determined by immunoblotting. Compared to S. typhimurium infection alone, pretreatment with probiotics and synbiotics induced 20 and 4 folds of miR 21 expressions, respectively. 